\title{
Goal-Oriented Requirements Engineering: State of the Art and Research Trend
}

\author{
Rosa Delima $^{1}$, Retantyo Wardoyo ${ }^{2}$, Khabib Mustofa ${ }^{3}$ \\ ${ }^{1,2,3}$ Departement of Computer Science \& Electronics, Universitas Gadjah Mada \\ ${ }^{I}$ Departement of Informatics, Universitas Kristen Duta Wacana \\ ${ }^{1}$ rosadelima@staff.ukdw.ac.id, ${ }^{2}$ rw@ugm.ac.id, ${ }^{3}$ khabib@ugm.ac.id
}

\begin{abstract}
The Goal-Oriented Requirements Engineering (GORE) is one approach that is widely used for the early stages of software development. This method continues to develop in the last three decades. In this paper, a literature study is conducted to determine the GORE state of the art. The study begins with a Systematic Literature Review (SLR) was conducted to determine the research trend in the last five years. This study reviewed 126 papers published from 2016 to 2020 . The research continues with the author's search for scientific articles about GORE. There are 26 authors who actively publish GORE research results. Twenty-six authors were grouped into seven groups based on their relation or coauthoring scientific articles. An in-depth study of each group resulted in a holistic mapping of GORE research. Based on the analysis, it is known that most research focuses on improving GORE for an automated and reliable $R E$ process, developing new models/frameworks/methods originating from GORE, and implementing GORE for the RE process. This paper contributes to a holistic mapping of the GORE approach. Through this study, it is known the various studies that are being carried out and research opportunities to increase automation in the entire RE process.
\end{abstract}

Keywords: goal-oriented modeling, GORE, requirements engineering, systematic literature review

\section{INTRODUCTION}

The goal-oriented approach has been widely used in research in the field of computers. Even the use of this approach has also been widely used in the field of software engineering and, more specifically, in the requirements engineering (RE) process. The application of the Goal-oriented RE (GORE) approach provides many advantages in the process of elicitation, analysis, specifications, and validation. This approach simplifies the process of defining requirements by users [1]. The GORE approach can be used to support the definition of functional and non-functional requirements on the system [2-3]. Various studies of the use of GORE to support process automation in RE have been carried out.
A literature study was conducted to find out the GORE state of the art over the last three decades.

This research is begins with SLR to determine the development of GORE research in the last five years. This paper contributes to a holistic mapping of the GORE approach in the three decades and the development trends of the GORE in the last five years.

The research continues with tracking authors on publications related to the Goal-oriented approach starting from the beginning; the approach was introduced and used in RE. Then continued in-depth studies of each group of authors on the GORE study. Based on this group of writers, a mapping of GORE publications was carried out which, were grouped into time periods and research categories in the RE field. Through holistic analysis, it can be seen that the state of the art Goal-oriented approach in RE.

The paper will be organized into four sections, such as the introduction in the first section, next in section 2, the paper will discuss research methodology. Then, section 3 is the result of the mapping along with the discussion, and the paper is closed with conclusion in section 4 .

\section{METHOD}

The topic of GORE papers for the last two decades can be seen in the Systematic Mapping Study (SMS) conducted by Horkoff et al. The SMS includes topic requirements engineering (RE), agents, reasoning, NFR or soft goals, adaptability, variability, or evolution, early RE, conflicts, scenarios, Business model or business intelligent, patterns, security, risk, or privacy, $\mathrm{Md}^{*}$, context, aspects, preferences, and compliance [4].

The publication of GORE articles has been widely carried out since 1998 until today. The main difference between SLRs that will be carried out is the period of paper reviewed. SLRs were carried out by [4-5] related to papers published between 1998 and 2015. This SLR will complement the SLR that has been done by reviewing articles published from 2016 until 2020. 
SLR was carried out with reference to the SLR process by [6-8]. SLR is done through a three-stage approach, such as concepts, studies, and results. The concept is a stage of formalization of issues through research questions that will be the basis for extracting information through the evidence gathered. In the second stage, studies are the stages of conducting a review by dissecting content, comparing, or linking parts of the existing evidence. Results from the studies will form the basis of the compilation results. At the results stage, the results of the analysis and synthesis of the data will be formulated into new data/knowledge, which will be formulated in conclusion. Based on these three stages, SLR has three main processes, such as planning, review execution, and result from the analysis [6].

Planning activities on the SLR include identifying requirements, formulating research questions, and developing review protocols. The review execution phase includes identification activities, selection of primary research, study quality assessment, data extraction, and monitoring. Review results activities include data analysis and data synthesis. The results will be displayed in a review report [7]. In the SLR, an analysis of the classification and mapping process of the collected data can be carried out, with reference to the three stages of the SLR; the steps of the systematic mapping study are formulated as in Fig. 1 [8]. The SLR process consists of 5 stages; there is the definition of research questions, conduct search, screening of papers, keywording using abstracts, and data extraction mapping process. The outcomes from each activity are review scope, all articles, relevant documents, classification schemes, and systematic maps, respectively.

\section{A. Research Questions}

The formulation of research questions (RQ) is the initial stage in the SLR. Specific RQ helps in giving direction and focus in the SLR process. There are four RQs formulated in GOM SLR, such as:

1) RQ1: How is the trend of GORE research in the last four years? Rationale: RQ1 aims to determine the development trends of GORE research. The trend is seen based on the number of publications each year and each type of GORE research contribution each year.

2) RQ2: What kind of contribution has been given to the GORE research that has been done? Rationale: RQ2 aims to determine the types of contributions provided by the paper reviewed. The contributions will provide information on how GORE is related to other methods/approaches for the automation and improvement of RE quality. This information is useful for knowing maps and opportunities for further GORE research.

3) RQ3: What types of systems have been developed through the application of GORE? Rationale: RQ3 aims to obtain information on what kind of system has implemented GORE in the RE process.

\section{B. Conduct Search Based on Keywords}

The search is done through some keywords, such as goal model requirement engineering. Based on the specified keywords, the literature search process is carried out. The investigation is carried out for publications from 2016 until 2020 in 3 literature sources such as Science Direct, Scopus, and IEEE. The search is done using advanced features, which means the specified keywords are in the title, abstract, or keywords. The search is focused on articles in the form of journals and proceedings.

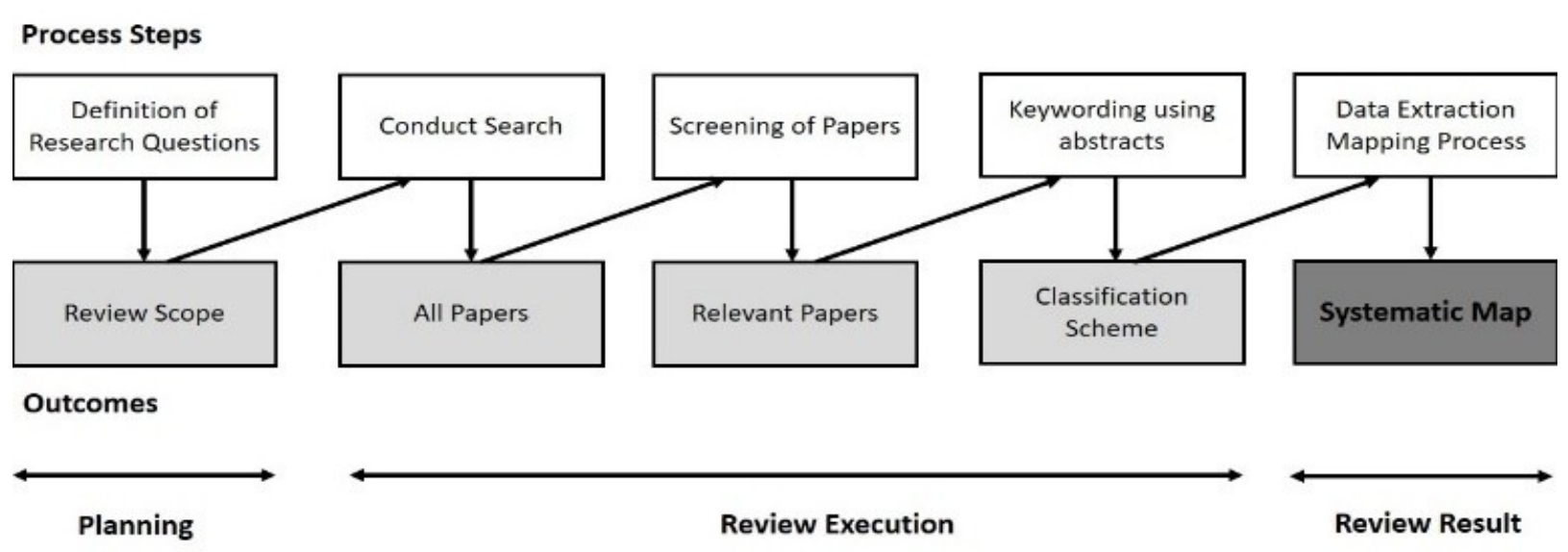

Fig. 1 Systematic Literature Review and Mapping Diagram [7-8] 


\section{Screening Paper and Keywording of Abstracts}

The search using keywords with the specification of journal articles has resulted in 414 papers, then a screening process was conducted on documents related to inclusion and exclusion factors. Inclusion and exclusion criteria are used to filter the document that is not relevant to RQ [8]. Inclusion is done to filter out the same document, and exclusion is done to screen irrelevant papers. For the exclusion factor, filtering is done through two stages, such as 1) examination of abstracts and keywords and 2) abstract reading by the author. The process of abstract examination is carried out by examining the existence of the word "goal model" or "goal-oriented" in the abstract. After passing the first examination, then an abstract reading was carried out by the author to assess the relevance of the paper. Based on the results of the screening, there were 126 relevant papers. Details of the filtered paper from each stage can be seen in Fig. 2. After screening papers, the next process is keywording. Keywording is a way to reduce time in classifying SLRs. Keywording is done through abstract reading and determining keywords to form classification schemes that are adapted to the research context [8]. The results of keywording are in the form of a classification scheme that consists of year classification and type of publication, contribution classification, and classification of system types.

\section{RESULTS AND DISCUSSION}

In this section, we will discuss the results and analysis of the SLR, which is arranged based on the defined RQ. The discussion of results is based on each RQ.

\section{A. RQ1: How is the trend of GORE research in the last} five years?

Based on the screening process, there have been 126 relevant papers. It was distributed in five years of publication, from 2016 to 2020 . The distribution number of papers based on a year in a sequence is such as 20 , $26,22,38$, and 20 . The paper was categorized into two parts, such as journal and proceeding. The graph of the distribution number of papers based on year and type of publication can be seen in fig. 3 .

Overall, the number of papers in journals and proceedings is almost balanced. The percentage of articles published in journals and proceedings was $47.6 \%$ and $52.4 \%$, respectively. The interesting thing in fig. 3 , it can be seen that the types of publications that dominate in 2017 and 2018 were proceeding, whereas, in 2019 and 2020, the publication is mostly done in journals.
Based on the graph in Fig. 3, it can be said that the GORE research trend can be said to be relatively stable with around twenty papers each year, and the trend shows that many research publications have been carried out in journals in the last two years.

\section{B. RQ2: What kind of contribution given through the GORE research done?}

After conducting an in-depth review, the contributed paper was grouped into six groups, namely Implementation (P), Method Development (I), study, and method evaluation (E), New Approach/ Methods/ Framework/ Model/ Language/ Technique/Algorithm $(\mathrm{N})$, and ongoing research (OR). The implementation category means that the paper is GORE implementation in various forms of systems, while method development is a paper that focuses on the development of GORE methods. New Approach means the application of GORE produces new approaches, methods, frameworks, models, language, techniques, or algorithms, and ongoing research means that GORE related research is still ongoing. Mapping contributions associated with the year of publication can be seen in Fig. 4(a).

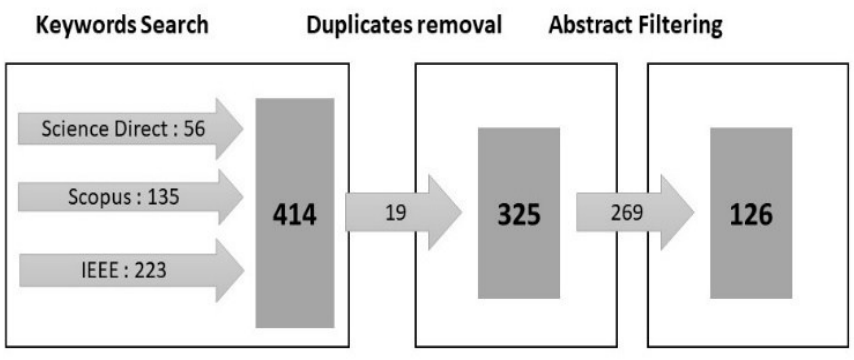

Fig. 2 Screening phases and number of selected papers

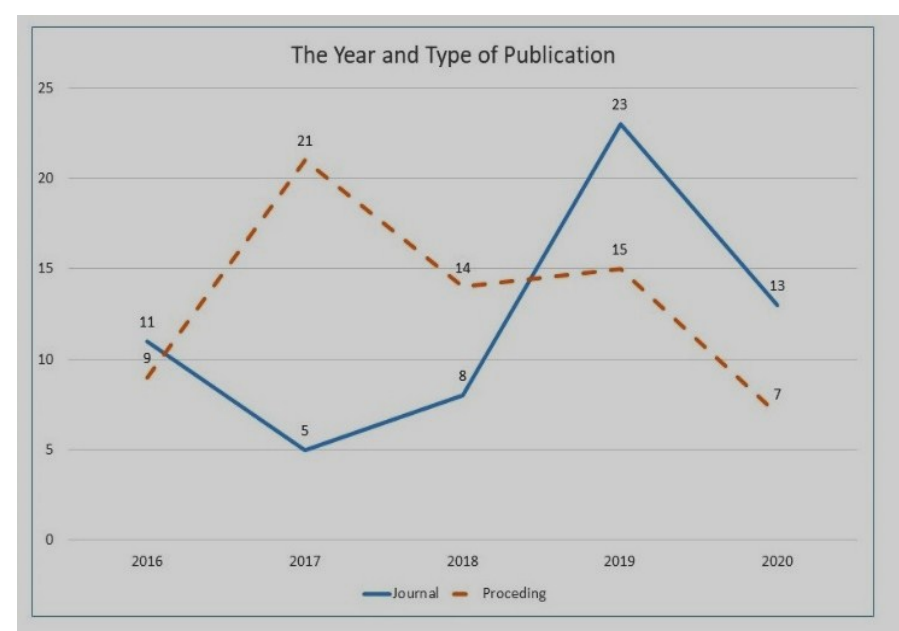

Fig. 3 Graph of the number of papers based on the year and type of publication 


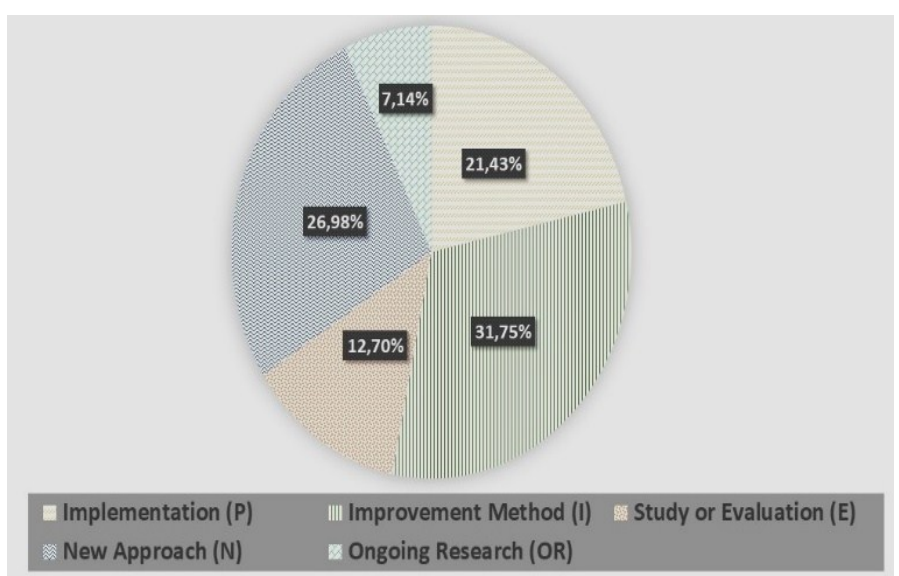

(a)

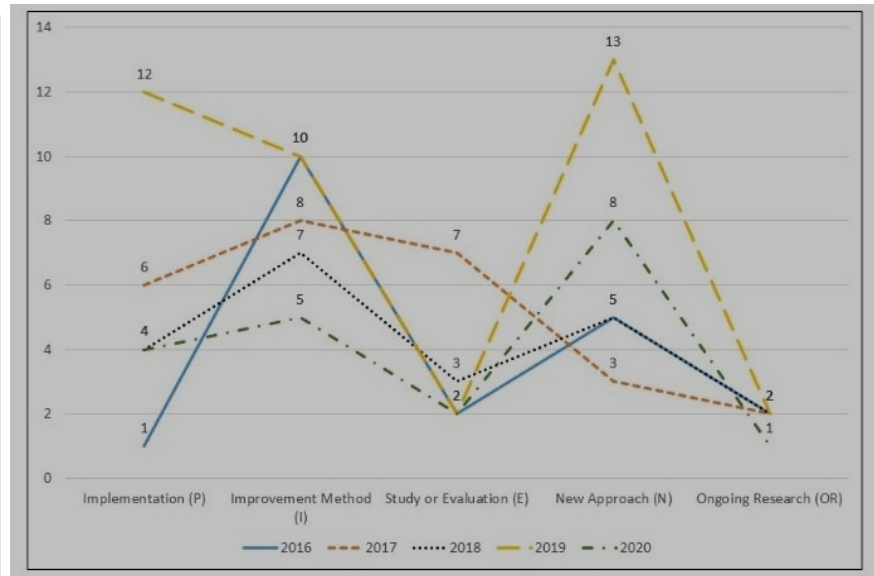

(b)

Fig. 4 Mapping chart based on contribution associated with the year of publication (a) and Graph of the number of papers based on contribution (b).

Based on Fig. 4(a), it is known that the contribution in 2016 until 2018 ware mostly on the improvement method, and 2019 until 2020 showed that the contribution was a new approach. If it is viewed from the type of contribution, the GORE implementation (P) was mostly conducted in 2019, while the paper that contributed to the most improvement GORE method (I) was in 2016 and 2019. Studying or evaluating the GORE method (E) was in 2017. The new approach (N) created from GORE was in 2019, and ongoing research (OR) shows a flat trend from 2016 to 2020 . Overall from 2016 to 2020 , it is known that as many as $31.75 \%$ of papers contributed to the improvement (I) GORE method, $26.98 \%$ of papers contributed at a new approach $(\mathrm{N}), 21.43 \%$ for GORE implementation $(\mathrm{P})$, $12.70 \%$ for studying and evaluation (E) GORE method, and $7.14 \%$ was ongoing research. The number of papers and their contributions can be seen in Fig. 4(b).

The improvement of the GORE method includes improvement in the modeling language, improvement approach or model on GORE, improvement on function or method to enhanced GORE's capability, and improve the GORE method to enhance the quality of RE modeling. The improvement of modeling language includes developing $i^{*}$ language and notation [9] [10], User Requirements Notation (URN) [11], Goal-oriented Requirements Language (GRL) [12], GORE modeling development uses Systems Modeling Language (SysML) [13], and Unified Modeling Language (UML) [14]. The improvement of approach or model on GORE includes Goal Model for Dynamic Systems (GmoDS) [15], Goal-Oriented Requirements Engineering framework for Systems of Systems (SoSGORE) [16], Agent Goal Model [17], Goal Dependency Model with
Objects (GDMO) [18], trust-aware goal modeling [19], and realistic goal models [20].

The development of function and method is often done on GORE. The capability development starts from planning stage, Requirements analysis, goal analysis, reasoning in goal, choosing goal, goal priorities, goal conflicts, goal consistency, goal dependability, goal decomposition, and goal repairing. The GORE method improvement is related to quality improvement including goal validation, goal quality, requirements satisfaction in goal model, and requirements visulization.

The third category is the study and evaluation of GORE. Study and evaluation are done to measure the effectivity of Knowledge Acquisition in the autOmated Specification (KAOS) method, the study on strengths of modeling on the GORE approach and possibility for automation process, and evaluation to find the gap between research and practice on the GORE method. Evaluation is also done to measure method effectivity at dealing with problems related to requirements evolution or changes, and method capability to support the efficiency of decision making. Study and evaluation are also done related to the diagrammatic notation issue on the goal model. The study includes diagram description, users' perception, users' understanding, tools to support method visualization, and architecture evaluation. Other studies are done related to the use of the GORE method to support specification on Model-Driven Development (MDD), and tool modeling on Model-Driven Engineering (MDE) . The evaluation was also carried out on the usability of the $\mathrm{i} *$ language, and the study of goal-oriented healthcare equipment regulations.

The development of the new function, method, model, framework, approach, and methodology is also a contribution to GORE research. Harkoff et al. [21] did 
research on the possibility to develop a creative goal model for elicitation, Busari and Letire [22] developed a RADAR, a modeling language and analysis tool, Anda [23] developed a mathematics function for adaptability of RE, and Aziz [24] developed control mechanism on Grids. Neace et al. and Rodrigues et al. developed new methods using the GORE Method, such as a method to measure the capability of RE [25] and a method to repair contextual variable specification using strategies for dependability [26]. Besides that, [27] introduces a method for modeling analyzing non-functional goals and [28] proposes a test scenario generation method using a KAOS-based approach. Besides the method, the GoPED algorithm was also developed from a Goal-oriented approach [29].

The new models developed using the GORE method include model-driven for IT governance, the model for alignment of the organizational characteristics, the model for trustworthiness requirements, and GORO.

The new frameworks developed to include the STSIQ framework, framework for dynamics requirements' reconfiguration, Tropos4AS framework, a goal-use case integration framework, privacy and trust requirements framework, Go-For framework, and Continual Value(s) Assessment (CVA) framework. Besides, the framework is also developed by combining GORE with Petri Nets.

The new approaches and methods developed using GORE Method are as psychologically-driven requirements engineering approach, autonomy requirements engineering approach, $M \& S$ methodology, an approach for goal-obstacle analysis, and the multiaspectual analysis approach. CARgo tools developed for contextual annotation and reconciliation and GoalCapability-Commitment based Executable Tree (GCCETree).

\section{RQ3: What types of systems have been developed through the application of GORE?}

GORE has been applied to various types of systems. Self-adaptive systems are the systems that use GORE mostly for the RE process. Adaptive RE is an important issue for developing systems that are able to adapt to changes in the environment and user requirements. In addition, Socio-Technical Systems, Systems of Systems, and Health Systems also often apply GORE. Other systems that implement $\mathrm{RE}$ include intelligent transportation systems, Software Product Lines, AgentBased Systems, Complex Systems, Cyber-physical Systems, Software Systems, Safety-Critical Systems, and Legacy Systems.

\section{GORE State of the art}

State of the art is compiled based on the author's search for GORE research articles sourced from Scopus. Based on the author's search for scientific articles, a list of authors who published more than nine articles from 2001 to mid-2019 was obtained, as shown in Fig. 5.

A review was also carried out on the relationship between authors in fig. 5. The analysis was carried out by searching for articles co-authored by GORE authors. The results of the analysis are in the form of author relations and the number of articles written together. The co-authoring relationship between GORE authors can be seen in fig. 6(a). In fig. 6(a), each author is represented in a node. Node numbering is based on the author's order in Fig. 5. This numbering also indicates the ranking of the author's number of publications. The connecting line between the authors states co-authoring, and the numbers on the links represent the number of co-authored publications between authors. The diagram in Fig. 6(a) is then summarized in several collaboration groups, as shown in Fig. 6(b). Fig. 6(b) describes there are seven groups of collaborative authors in writing published articles.

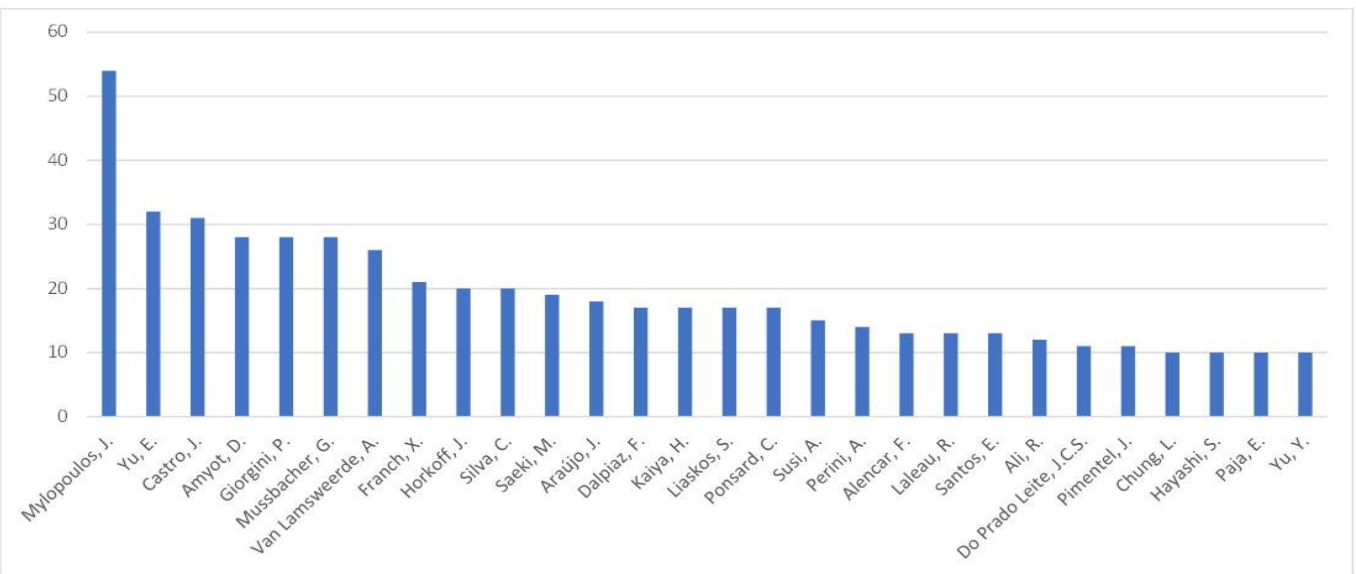

Fig. 5 GORE publications by the author from 2001 to mid-2019 with a number of publications $>9$ 


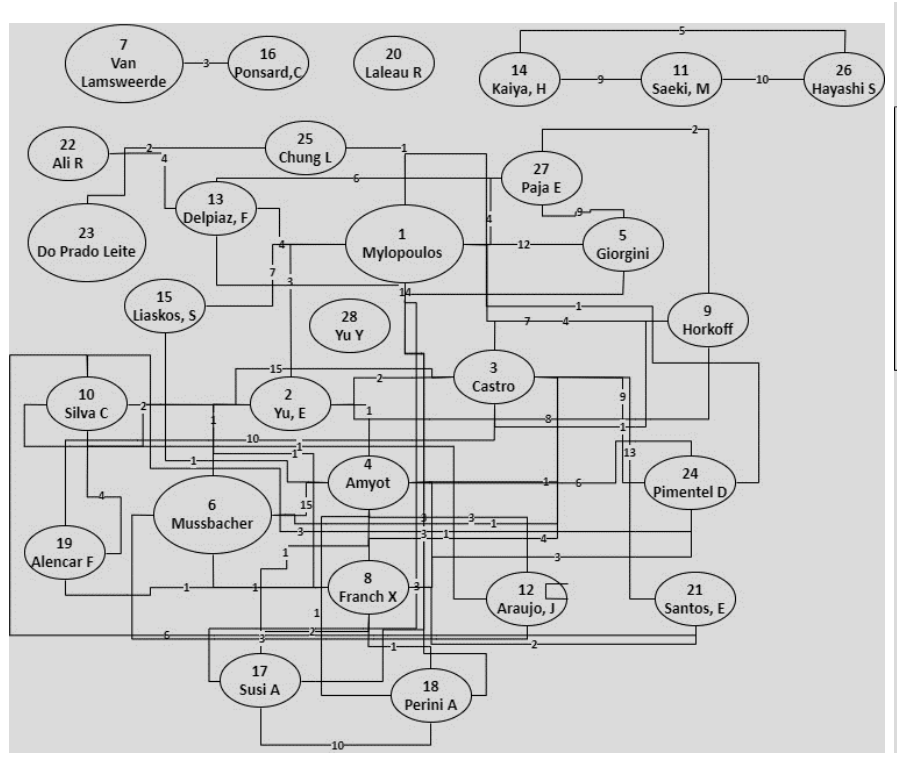

(a)

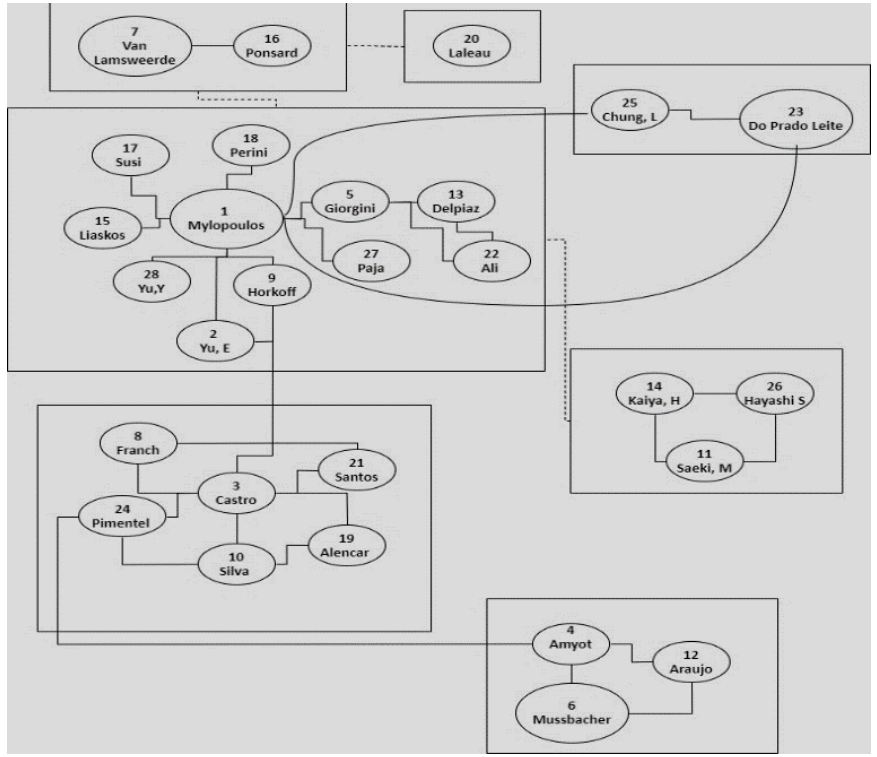

(b)

Fig. 6 Relationship diagram of the publication with the authors (a) and a collaborative group of GORE publications authors (b)

Sequence analysis was carried out to get information related to the author of the article in Fig. 6(b). The analysis is done based on the year of publication. Based on this analysis, it is known that the development of the GORE method was initiated by Robinson [30], who published his research in 1989. The goal approach is used to integrate specifications and deal with conflicts of requirements originating from multiple users [30].

Based on the GORE author/researcher grouping in Fig. 6(b), an in-depth analysis was carried out to determine the research publication journey of each author group. The first author group is the Axel Van Lamsweerde group. Van Lamsweerde is the person who introduced the term GORE for Goal-Oriented Requirements Engineering. As seen in fig. 6, Axel van Lamsweerde collaborated with C. Ponsard and other authors such as Anne Dardenne, Robert Darimont, and Emmanuel Letier to write articles related to GORE. They develop GORE starting from requirements elicitation, developing the GRAIL/KAOS environment framework for GORE, conflict management, and obstacles to GORE, GORE agents, goal satisfaction, and verification, and validation issues at GORE.

The publication by Axel Van Lamsweerde and the team began in 1991 with the application of a goaldirected concept for requirements acquisition. Publications related to this continued in 1993. For requirements analysis/reasoning, Van Lamsweerde and team published research related to goal divergent and goal satisfaction. Another publication was carried out related to goal-directed elaboration from 1995 to 2002 . For goal specifications, Van Lamsweerde published research related to event-based specification and goal animation. In the period between 2006 and 2015, publications were carried out related to requirements quality for verification, validation, and goal compliance. Publications related to goal compliance are closely related to the publication of goal obstacle which was implemented in 2000, 2012, and 2017. Publications related to security requirements were also carried out by this team from 2001 to 2006. In addition, Van Lamsweerde and the team also published their research using a formal approach to goal refinement. For riskbased RE, this team publishes an article related to uncertainty and exception handling in requirements. There are two frameworks published by the Van Lamsweerde team, namely KAOS and reuse requirements. The team also publishes on the integration of the goal-directed approach with the scenario and state machine approach in a synthetic model.

The second group of authors on the GORE article is the group initiated by John Mylopoulos. This group is a collaborative author group with the most number of authors. Mylopoulos, as in fig. 6(b), collaborates with, among others, Eric Yu, Paolo Georgini, Jennifer Horkoff, and Sotirios Liaskos. This second group of authors has published a lot on requirements analysis, variability, security, validation, evolution, risks, and automatic requirements. 
Mylopoulos and the team did an initial publication related to goal-oriented concepts, which began in 1996, followed by an article on the shift from object-oriented to goal-oriented paradigm. For requirements acquisition, Mylopoulos published research related to the organizational structure approach as system architecture and the gamification approach to simplify the requirements elicitation process in organizations. Mylopoulos team published model checking, requirements traceability, formal and aspect-oriented approaches to requirements analysis/reasoning from 2001 to 2005. Publications on requirements traceability and aspect-oriented variability analysis were still carried out from 2006 to 2010. Over time, publications related to requirements analysis/reasoning includes the use of decision-theoretical approaches, knowledge representation, and mining software analysis. Between 2016 and 2020, requirements analysis/reasoning related to similarity-based reasoning and the AGON Framework. Agon framework is a framework used for needs analysis and design with a gamification approach.

Mylopoulos and team conducted initial publications related to goal-oriented concepts, which began in 1996, followed by articles on publications related to goal refinement related to refinement through the anti-goals approach and refinement with calculus. For requirements specifications, publications focused on formal specification and security mechanisms in 2015 . Publications related to requirements quality were carried out by the Mylopoulos team with a multi-party agreement and annotating for goal satisfaction. A multiparty agreement is used to validate and check the acceptability conditions of the requirements.

Publications for risk and reuse requirements were carried out by the Mylopoulos Team between 2011 and 2015. Publications related to evolution requirements are mostly carried out using the reverse engineering approach, reconfiguration requirements, self-repair, and self-adaptive requirements. Publications on security, trust, and privacy requirements are also mostly carried out by the Mylopoulos Team, covering issues related to security, trust and privacy, ST-Tool development, SI * modeling, and the MUSER framework for security requirements. In addition, Mylopoulos and the team also published a lot of their research related to nonfunctional requirements (NFR) using a goal-oriented approach.

In their publication, Mylopoulos and the team use various approaches to support GORE, namely the formal approach, agent and multiple agent approaches, ontology approaches, aspect-oriented approaches, and business process modeling. Meanwhile, for system application, most of the publications focus on Law compliant Systems, Adaptive Systems, Socio-Technical Systems, and mobile systems.

There are two frameworks developed by Mylopoulos and the team for GORE, namely the $i$ * framework and the Tropos framework. Both of these frameworks continue to be developed by adding to the functions and capabilities of the framework.

Still, in direct contact with the second group, the third group includes Jaelson Castro, Carla Silva, Emanuel Santos, Joao Pimentel, and Franch Xavier. They mostly do publications related to the development of the $i^{*}$ meta-model, goal alignment, requirements changes, software product lines, and adaptive system requirements. They publish papers starting with Goaloriented concepts, which deal with filling the gap between organization requirements and object-oriented models. For requirements elicitation, the publication discusses requirements elicitation and formalization, then continued with requirements change in 2012, and gamification in elicitation in 2018. For requirements analysis, the publication focuses on issues of traceability, variability, and reasoning using an agent.

Publications related to the requirements specification were carried out by Castro and the team, starting with the architectural model derived from software requirements. In the period 2011 to 2015, they published the STREAM method and its development for deriving architecture from the requirements model. In addition, this period also published the JGOOSE tool for architecture modeling with use cases.

Non-Functional Requirements (NFR) is one category that has a lot of publications on this team. The publication began with the application of the COTS (Commercial-Off-The-Shelf) method and the formal approach to NFR in 2001. The publication then continued with the development of the AoRE (Aspectoriented RE) method, soft goals in a use case, variability, and adaptability as variables in NFR.

Castro and the team also published a requirements document, which they named AIRDoc in 2008. This document was developed with a goal-oriented approach. Then the document was developed using Goal Question Metrics in a publication in 2011. Castro and the team also published a publication related to the application of a formal, agent and multi-agent approach, and aspectoriented in goal-oriented.

This team is developing i* model previously created by Mylopoulos and the team, including developing the iSTAR language for the GORE approach. In addition, they also developed the TROPOS framework by adding an architectural design and multi-agent system to TROPOS. 
In the last decade, 2011-2020, Castro and the team used a goal-oriented approach to the development of adaptive systems, Socio-technical Systems, Software Product Lines, Context-sensitive systems, and SafetyCritical Systems. In 2019 the SARSSI * method was published for safety requirements specification. This method combines STAMP / STPA (Systems-Theoretic Accident Model and Processes) and $i *$ language [31].

The two next group consists of three authors referring to fig. 6. The first group consists of Daniel Amyot, Gunter Mussbacher, and Joao Araujo. They published articles related to Aspect-Oriented Goal Models, business process compliance, goal model analysis, i* implementation, and Goal Requirements Language (GRL). The second group consisted of Motoshi Saeki, Haruhiko Kaiya, and Shinpei Hayashi. They published research on Attributed Goal-Oriented Requirements Analysis (AGORA), Ontology-based requirements analysis, Goal-oriented and ontology requirements elicitation (GOORE), and requirements change.

These team started publication on the goal-oriented model in 1998 with a multi-agent and feature interaction in the goal model. Then they published a paper related to the User Requirements Notation (URN) model, which was followed by Goal Requirements Language (GRL). GRL is the development of URN. Publications related to URN and GRL dominate the publications conducted by Amyot's team. Apart from URN and GRL, they also published research related to Use Case Maps (UCM).

Publications related to security, trust, and privacy in requirements were carried out by Amyot and his team from 2007 to 2014, starting with the legal perspective in RE, Privacy compliance, business process compliance, and legal GRL. The aspect-oriented approach is used by Amyot and the team for the development of URN, GRL, and BPM models. Besides that, publications are also carried out related to the development of an aspectoriented approach by integrating with other approaches such as semantic-based, goal, and scenario. Aspectoriented is also used to perform RE on software product lines.

In their publication, Amyot and the team integrated the goal model with other approaches, namely rulebased, activity theory, relaxation based on structural patterns, and arithmetic semantic of features. In addition, they also published research on business intelligence for adaptive systems, URN and Regulator compliance in SPL, and RE for health systems. For requirements quality, Amyot and team published RE validation using a questionary based in 2016 and conflict resolution in goal model in 2017.

Most of the publications conducted by Motoshi Saeki and the team are research related to Goal-oriented requirements analysis (GORA). Publications related to this model were carried out from 2002 until publication in 2017. In the second decade, Saeki and Team also published research related to Goal-oriented idea generation methods for requirements elicitation in 2003 and goal-oriented and ontology in 2007. In the third decade, this team published research related to multidimensional goal refinement in GORE in 2015 and requirements analysis for Socio-technical system in 2016.

The last two groups are the authors Regine Laleau and Julio Cesar Sampaio do Prado Leite. Laleau and her team published research related to the development of the KAOS Goal Model, a formal meta-model for formal RE, Event B formal meta-model on KAOS, and uncertainty in RE. The group of do Prado Leite and Lawrence Chung published research related to NonFunctional Requirements (NFR), requirements validation, and requirements conceptual acquisition. Julio Cesar Sampaio do Prado Leite and the team published their research related to the analysis of variability and high variability in goal models. For requirements validation, they published research on RETR from legacy code in 2005. This team did many publications related to NFR in RE starting in 2003 with a soft goal for NFR, followed by the use of Fuzzy logic in 2011 and accessibility requirements as part of the NFR in 2016. This publication related to the team's goal model and aspect-oriented was started with discovering aspects from requirements goal models in 2004. Other publications related to aspect-oriented were conducted in 2006, 2009, 2011, and 2013.

I* framework development was also carried out by do Prado Leite and the team. This is known through their publications, which include assessment in $i^{*}$, an extended lexicon in $\mathrm{i}$ *, ERi * c Method, verifying in $\mathrm{i}^{*}$, and traceability in $i^{*}$. Publications related to the agent and multi-agent approach were carried out by do Prado Leite and team to develop an integration model between scenario, $i^{*}$, and aspectT. In addition, they also developed the AGFL and agent system driven method for intentional agents code in the $i^{*}$ architecture model.

In this paper, a SLR has been conducted to find out the development of GORE research in the last five years (2016 to 2020). This SLR was carried out as a continuation of the SLR carried out by Horkoff et al. [5]. SLR produced 126 relevant papers published from 2016 to 2020. The articles were sourced from the Scopus, Science direct, and IEEE database. The distribution of the number of papers per year ranges from 20 to 38 papers. The analysis was carried out by categorizing the article contributions. There are six categories, namely Implementation (P), Method Development (I), study and 
method evaluation (E), New Approach/Methods/ Framework/Model/Language/Technique/Algorithm (N), and ongoing research (OR). Based on the analysis, it is known that more than $50 \%$ or $58.73 \%$ of the papers contributed to the development of the GORE method and the development of new approaches/models/frameworks/tools at GORE. A fairly large contribution of $21.43 \%$ is on the implementation of the GORE method for the development of various systems. Self-adaptive systems have become a form of system that has implemented a lot of GORE in the last three years. This is very closely related to the need for a requirements engineering development process that is adaptive to changing user requirements.

This paper also analyzes the development of the GORE approach over the last 30 years. The analysis was carried out based on a literature search regarding authors of scientific articles for GORE. There are 26 dominant authors in GORE publications. The analysis was carried out on the relationship between the authors in the article. Through this analysis, we obtained seven groups of authors. Furthermore, the method/topic studied in the publication is carried out. Based on this analysis, the GORE State of the art was produced from its early development in the 1990 s to 2020 .

\section{CONCLUSION}

The information obtained from the SLR shows that GORE is a reliable method to use for the RE process, but there are still some issues that must be resolved in order to automate the RE process. There are several issues that can contribute to increasing the automation capability of the RE process. The first issue is related to development of GORE standard input forms in the elicitation stage. The standard input form is the basis for obtaining data from the user's requirements. Formulating user input standards at GORE will contribute to the elicitation stage in RE. The issue of analysis to detect conflict goals from multiple users and conflict handling at GORE still requires the contribution of implementing various methods, both in the form of a decision support system and an intelligent system, so that this process is fully automated with minimal involvement of system analysts. The issue of developing the GORE knowledge base also still requires contributions from researchers so that agents at GORE can make inferences for automatic requirements analysis and validation.

\section{ACKNOWLEDGMENT}

Special thanks to Computer Science and Electronics Department Universitas Gadjah Mada and Faculty of Information Technology Universitas Kristen Duta
Wacana for providing facilities and funding for publishing this article.

\section{REFERENCES}

[1] C. M. Nguyen, R. Sebastiani, P. Giorgini, and J. Mylopoulos, "Multi-objective reasoning with constrained goal models," Requir. Eng., vol. 23, no. 2, pp. 189-225, 2018, doi: 10.1007/s00766-016-0263-5.

[2] J. Horkoff, N. A. Maiden, and D. Asboth, "Creative goal modeling for innovative requirements," Inf. Softw. Technol., pp. 1-16, 2018, doi: 10.1016/j.infsof.2018.09.005.

[3] C. Kalloniatis, "Incorporating privacy in the design of cloud-based systems: a conceptual meta-model," Inf. Comput. Secur., vol. 25 No.5, pp. 614-633, 2017, doi: 10.1108/ICS-06-2016-0044.

[4] J. Horkoff et al., "Goal-oriented requirements engineering: an extended systematic mapping study," Requir. Eng., vol. 24, no. 2, pp. 133-160, 2019, doi: 10.1007/s00766-017-0280-z.

[5] J. Horkoff et al., "Goal-Oriented Requirements Engineering: A Systematic Literature Map," in Proceedings - 2016 IEEE 24th International Requirements Engineering Conference, RE 2016, 2016, pp. 106-115, doi: 10.1109/RE.2016.41.

[6] J. Biolchini, P. G. Mian, A. C. C. Natali, and G. Travassos, "Systematic Review in Software Engineering," Ri de Janeiro, 2005. doi: 10.1007/978-3540-70621-2.

[7] B. Kitchenham, "Guidelines for performing Systematic Literature Reviews in Software Engineering," 2007. doi: 10.1145/1134285.1134500.

[8] K. Petersen, R. Feldt, S. Mujtaba, and M. Mattsson, "Systematic Mapping Studies in Software Engineering," in Proceedings of the 12th international conference on Evaluation and Assessment in Software Engineering, 2007, vol. 80, no. 2, pp. 68-77, doi: 10.1142/S0218194007003112.

[9] L. López, X. Franch, and J. Marco, "Specialization in the iStar2.0 Language," IEEE Access, vol. 7, pp. 146005-146023, 2019, doi: 10.1109/ACCESS.2019.2940094.

[10] E. Gonçalves, J. Araujo, and J. Castro, "PRISE: A process to support iStar extensions," J. Syst. Softw., vol. 168, pp. 1-33, 2020, doi: 10.1016/j.jss.2020.110649.

[11] R. Saini, S. Bali, and G. Mussbacher, "Towards web collaborative modelling for the user requirements notation using eclipse che and theia IDE," in Proceedings - 2019 IEEE/ACM 11th International Workshop on Modelling in Software Engineering, MiSE 2019, 2019, pp. 15-18, doi: 10.1109/MiSE.2019.00010.

[12] S. Sartoli, S. Ghanavati, and A. Siami Namin, "Towards Variability-Aware Legal-GRL Framework for Modeling 
Compliance Requirements," in 2020 IEEE 7th International Workshop on Evolving Security \& Privacy Requirements Engineering (ESPRE) Towards, 2020, pp. 7-12, doi: 10.1109/espre51200.2020.00007.

[13] D. Amyot, A. A. Anda, M. Baslyman, L. Lessard, and J. M. Bruel, "Towards Improved Requirements Engineering with SysML and the User Requirements Notation," in Proceedings - 2016 IEEE 24th International Requirements Engineering Conference, RE 2016, 2016, pp. 329-334, doi: 10.1109/RE.2016.58.

[14] H. Kaiya and K. Haga, "A CASE tool for Goal Dependency Model with Attributes based on An Existing UML Editor," in Procedia Computer Science, 2017, vol. 112, pp. 1196-1205, doi: 10.1016/j.procs.2017.08.033.

[15] M. Brezovan, L. Stanescu, and E. Ganea, "Expressing GMoDS models into object-oriented models using the event-B language," Inform., vol. 40, pp. 29-42, 2016.

[16] S. Alhajhassan, M. Odeh, and S. Green, "Aligning systems of systems engineering with goal-oriented approaches using the i* framework," in ISSE 2016 2016 International Symposium on Systems Engineering - Proceedings Papers, 2016, pp. 1-7, doi: 10.1109/SysEng.2016.7753125.

[17] I. Vistbakka and E. Troubitsyna, "Pattern-Based GoalOriented Development of Fault -Tolerant MAS in Event-B," in 18th International Conference, PAAMS 2020, 2020, pp. 327-339.

[18] H. Kaiya, "Modelling Goal Dependencies and Domain Model Together," in Procedia Computer Science, 2016, vol. 96, pp. 791-800, doi: 10.1016/j.procs.2016.08.242.

[19] H. C. Lee, M. J. Kim, M. Shehab, and S. W. Lee, "Trust-Aware Goal Modeling from Use Case for Cooperative Self-Adaptive Systems," in Proceedings 2018 IEEE International Conference on Systems, Man, and Cybernetics, SMC 2018, 2018, pp. 4405-4410, doi: 10.1109/SMC.2018.00744.

[20] T. Gunes and F. B. Aydemir, "Automated Goal Model Extraction from User Stories Using NLP," in 2020 IEEE 28th International Requirements Engineering Conference (RE) Automated, 2020, pp. 382-387, doi: 10.1109/re48521.2020.00052.

[21] J. Horkoff, N. A. Maiden, and D. Asboth, "Creative goal modeling for innovative requirements," Inf. Softw. Technol., vol. 106, pp. 85-100, 2019, doi: 10.1016/j.infsof.2018.09.005.

[22] S. A. Busari and E. Letier, "RADAR: A Lightweight Tool for Requirements and Architecture Decision Analysis," in Proceedings - 2017 IEEE/ACM 39th
International Conference on Software Engineering, ICSE 2017, 2017, pp. 552-562, doi: 10.1109/ICSE.2017.57.

[23] A. A. Anda, "Modeling adaptive socio-cyber-physical systems with goals and SysML," in Proceedings - 2018 IEEE 26th International Requirements Engineering Conference, $R E$ 2018, 2018, pp. 442-447, doi: 10.1109/RE.2018.00059.

[24] B. Aziz, "Modelling Fine-Grained Access Control Policies in Grids," J. Grid Comput., vol. 14, no. 3, pp. 477-493, 2016, doi: 10.1007/s10723-015-9351-x.

[25] K. Neace, R. Roncace, and P. Fomin, "Goal model analysis of autonomy requirements for Unmanned Aircraft Systems," Requir. Eng., vol. 23, no. 4, pp. 509555, 2018, doi: 10.1007/s00766-017-0278-6.

[26] A. Rodrigues, G. N. Rodrigues, A. Knauss, R. Ali, and H. Andrade, "Enhancing context specifications for dependable adaptive systems: A data mining approach," Inf. Softw. Technol., vol. 112, pp. 115-131, 2019, doi: 10.1016/j.infsof.2019.04.011.

[27] B. DeVries and B. H. C. Cheng, "Goal-Based Modeling and Analysis of Non-Functional Requirements," in Proceedings - 2019 ACM/IEEE 22nd International Conference on Model Driven Engineering Languages and Systems, MODELS 2019, 2019, pp. 261-271, doi: 10.1109/MODELS.2019.00010.

[28] T. Fujikura and R. Kurachi, "A Test Scenario Generation Method for High Requirement Coverage by using KAOS Method," in Proceedings - Companion of the 19th IEEE International Conference on Software Quality, Reliability and Security, QRS-C 2019, 2019, no. 2, pp. 542-543, doi: 10.1109/QRS-C.2019.00115.

[29] M. Ghasemi and D. Amyot, "Data preprocessing for goal-oriented process discovery," in Proceedings - 2019 IEEE 27th International Requirements Engineering Conference Workshops, REW 2019, 2019, pp. 200-206, doi: 10.1109/REW.2019.00041.

[30] N. Robinson, "Integrating Multiple Domain Specifications Goals," in Proceedings of the 5th international workshop on Software specification and design (IWSSD) '89, 1989, pp. 219-226.

[31] J. Vilela, C. Silva, J. Castro, L. E. G. Martins, and T. Gorschek, "SARSSi*: a Safety Requirements Specification Method based on STAMP/STPA and i* language," in BWare 2019: I Brazilian Workshop on lArge-scale cRitical systEms, 2019, no. September, pp. 17-24, doi: 10.5753/bware.2019.7504. 